\title{
Theta synchronization in the limbic system: the role of Gudden's tegmental nuclei
}

\author{
Bernat Kocsis, ${ }^{1-3}$ Gonzalo Viana Di Prisco ${ }^{3}$ and Robert P. Vertes ${ }^{3}$ \\ ${ }^{1}$ Laboratory of Neurophysiology, Department of Psychiatry, Harvard Medical School, 74 Fenwood Rd, Boston, MA 02115, USA \\ ${ }^{2}$ National Institute of Neurosurgery, Budapest, Hungary \\ ${ }^{3}$ Center for Complex Systems, Florida Atlantic University, Boca Raton, FL, USA
}

Keywords: behavioural state control, electroencephalogram, hippocampus, mammillary body, neural oscillators, rat, theta rhythm

\begin{abstract}
Theta rhythm is most prominent in the hippocampus but has also been recorded in other cortical and limbic structures and can play an important role in functional coupling of widely separated structures responsible for different components of the memory building process. Here we demonstrate in the rat that neuronal activity exhibiting strong state-dependent synchrony with rhythmic hippocampal electroencephalogram is present also at the brainstem level, specifically in the relatively small tegmental nuclei of Gudden intimately connected with the limbic forebrain. We found that during theta states, either occurring spontaneously or triggered by sensory stimulation in the urethane anaesthetized rat, all neurons in the anterior and ventral tegmental nuclei exhibited a consistent switch from irregular discharges to rhythmic bursts. The switch between these patterns closely matched the analogous transformations in the hippocampal EEG, but the level of synchrony between the two signals varied depending on the level of theta activation. During sensory stimulation, when theta is faster and more regular, the rhythmic bursts in the tegmentum showed extremely high coherence (up to 0.96) with hippocampal field potentials. During spontaneous theta, the average coherence was lower but still highly significant (0.62). Gudden's nuclei are reciprocally connected to the mammillary body complex (MB) occupying a strategic position at the gateway of hippocampofugal connections organized in the Papez circuit. Thus, coupling between the MB-Gudden circuit and the hippocampus and consequently the neuronal traffic through the Papez circuit and hence the assembly of limbic structures connected to the hippocampus may vary according to the activity in these specific brainstem nuclei.
\end{abstract}

\section{Introduction}

The role of the hippocampus in memory is well established and the importance of subcortical structures connected to the hippocampus has been known since the pioneering work of Korsakoff (1887) and Wernicke (1881). Although certain memory deficits are best described in the framework of a diencephalo-hippocampal syndrome (Mair et al., 1979; Victor et al., 1989; Squire et al., 1990; Tulving \& Markowitsch, 1997), virtually nothing is known about how these forebrain systems interact with each other and how their interaction is organized by the brainstem so as to account for the robust statedependent changes underlying learning and memory.

Rhythmic synchronization of neuronal activity is one possible mechanism which has been found to be critical for the control of functional coupling in many different systems of the brain. In the hippocampus, theta rhythmicity dominates the electroencephalogram (EEG) selectively during awake exploration and paradoxical sleep (Vanderwolf, 1969), and the direct relevance of this pattern of activity for the function of hippocampal circuits in memory is commonly acknowledged (Winson, 1978). Theta is also present in a number of subcortical structures some of which (e.g. medial septum, MS, Petsche et al., 1962; supramammillary nucleus, Kirk \&

Correspondence: Dr Bernat Kocsis, as above.

E-mail: bkocsis@hms.harvard.edu

Received 17 August 2000, revised 31 October 2000, accepted 2 November 2000
McNaughton, 1991; Kocsis \& Vertes, 1994; Bland et al., 1995) are positioned along the ascending multisynaptic pathway connecting the brainstem with the hippocampus and may directly contribute to the control of hippocampal activity (Vertes \& Kocsis, 1997).

Signs of multiple coupled theta oscillators have also been observed on the hippocampofugal side. Rhythmic neuronal activity was recorded in a number of structures which do not belong to the ascending brainstem-hippocampal axis but are more likely to receive direct or indirect rhythmic input from the septohippocampal system. The gateway to these limbic structures is represented by the mammillary body (MB) nuclei, one of the primary subcortical targets of the hippocampal formation (Swanson \& Cowan, 1975; Risold \& Swanson, 1996). The MB also receives massive inputs from the entorhinal cortex (Shibata, 1989) and MS (Swanson \& Cowan, 1979), and therefore theta-related activity may reach the MB via several routes. Neurons in the $\mathrm{MB}$ are capable of generating intrinsic membrane oscillations (Alonso \& Llinas, 1992; Llinas \& Alonso, 1992) and their discharge during theta states is synchronized with hippocampal EEG (Komisaruk, 1970; Kocsis \& Vertes, 1994; Bland et al., 1995). The MB is functionally closely associated with the hippocampus with respect to, for instance, a common involvement in memory (Victor et al., 1989; Sziklas \& Petrides, 1998) and spatial navigation (Blair et al., 1998; Stackman \& Taube, 1998), but the specific details of their co-operation are unknown. Anatomically, the MB supplies the hippocampal formation with channels to the limbic 
forebrain via Papez's circuit and to the 'limbic midbrain' (Nauta, 1958) via the mammillotegmental tract (Petrovicky, 1973; Allen \& Hopkins, 1990; Hayakawa \& Zyo, 1990b). This latter pathway also represents the descending branch of a strong reciprocal link between the MB and tegmental nuclei of Gudden. The ascending branch is the mammillary peduncle (Petrovicky, 1973; Allen \& Hopkins, 1989; Hayakawa \& Zyo, 1991), the route by which the activity of MB neurons and ultimately the limbic-bound hippocampal output can be specifically regulated. Their strategic anatomical position notwithstanding, almost nothing is known about the physiology of the tegmental nuclei of Gudden. Anatomical data indicate that these neurons send a GABAergic projection to MB (Allen \& Hopkins, 1989; Hayakawa \& Zyo, 1991; Wirtshafter \& Stratford, 1993), whereas the mammillotegmental projection is excitatory (Allen \& Hopkins, 1990; Hayakawa \& Zyo, 1990b). Reciprocal excitatoryinhibitory circuits are known to generate rhythmic oscillations in other parts of the brain (Contreras \& Steriade, 1996; Contreras et al., 1996; Ritz \& Sejnowski, 1997; Llinas et al., 1999; Plenz \& Kitai, 1999).

The aim of this study was to investigate the neuronal activity in the tegmental nuclei of Gudden and its relationship to the hippocampal EEG. Based on the intrinsic pacemaking properties of MB neurons (Alonso \& Llinas, 1992; Llinas \& Alonso, 1992) and the neurochemical construction of the MB-tegmental connections (i.e. excitatory in one direction and mainly inhibitory in the other, Petrovicky, 1973; Allen \& Hopkins, 1989; Wirtshafter \& Stratford, 1993) we hypothesized that rhythmic activity may be maintained in the MB-tegmental loop and this rhythm may exhibit a certain level of autonomy from the septohippocampal generator. If this proves to be the case, coupling between this circuit and the hippocampus, and consequently the neuronal traffic through Papez's circuit and hence the assembly of limbic structures connected to the hippocampus may vary according to the activity in these specific brainstem nuclei. Thus, through the Gudden nuclei, information available on the brainstem level could be integrated into the activity of the limbic system. Some of these data have been published in abstract form (Kocsis, 1999).

\section{Materials and methods}

Experiments were performed on 33 Sprague-Dawley rats under urethane anaesthesia (1.2-1.5 g/kg, i.p.) under guidelines of the Florida Atlantic University Institutional Animal Care and Use Committee. For EEG recording, two Teflon-coated stainless steel wires (diameter $125 \mu \mathrm{m}$ ) were implanted $(3.5 \mathrm{~mm}$ posterior to the bregma, $2.2 \mathrm{~mm}$ from midline), one in the CA1 region the other immediately below the hippocampal fissure, and fixed to the skull with dental acrylic. Hippocampal field potentials were recorded monopolarly relative to a common indifferent electrode located anterior to the bregma, with bandpass filter settings of $1.5-75 \mathrm{~Hz}$. The extracellular unit electrode (3-7 MOhm tungsten or 4-6 MOhm glass pipette filled with $0.5 \mathrm{M}$ sodium acetate) was lowered slowly through a hole with a diameter of 1.5-2 mm centred $0.5 \mathrm{~mm}$ anterior to the lambda. In some experiments the electrode was lowered at several locations within a small area until a theta rhythmic signal was identified on the audio monitor. The unit recording was filtered between $300 \mathrm{~Hz}$ and $10 \mathrm{kHz}$ and stored on magnetic tape and analysed off-line. Each neuron was recorded during control, associated with low amplitude irregular activity in the hippocampus, and at least during one theta episode which occurred either spontaneously or in response to sensory stimulation (tail pinch). The location of the recorded neurons was marked by passing DC current through the electrode. At the end of experiments, the rats were perfused intracardially with saline and $10 \%$ formalin and processed histologically to reveal the location of the electrode marker in the brain.

A total of 89 theta segments were analysed (27 spontaneous, 62 sensory elicited); 1-5 such segments in each neuronal recording. Rhythmicity in the unit activity and its correlation with hippocampal theta was analysed both in the time and frequency domains, as described previously in detail (Kocsis \& Vertes, 1992; Kocsis \& Vertes, 1994). Analogue-to-digital conversion was performed with 12-bit resolution at two different sampling rates (using the ISC-16 A/ D card and EGAA software, RC Electronics, Santa Barbara, CA, USA). The spike train from single cell recordings with high signal-tonoise ratio was processed with a slope/height window discriminator and together with the other signals was sampled at $250 \mathrm{~Hz}$. For separation of spikes in multiunit extracellular recordings or in noisy recordings, the signals were sampled with sampling period between 30 and $60 \mu$ s. Time domain techniques provided detailed information on the dominant rhythmic component of the signals and their temporal dynamics. Frequency domain analysis, which extends over a wide range of frequencies including the theta frequency, was used for statistical testing of the significant relationship between EEG and unit signals. Time domain analysis included autocorrelograms and unitEEG cross-correlogram (spike-triggered averaging) performed using the EGAA software (RC Electronics) and time frequency analysis using Origin 5.0 (OriginLab, Northampton, MA, USA). In the frequency domain, autospectra for both the unit and EEG signals along with their coherence function were calculated for 10-25 contiguous windows of $4 \mathrm{~s}$ duration, using the fast Fourier transform (FFT) analysis implemented in a customized program (Kocsis \& Vertes, 1992; Kocsis \& Vertes, 1994). The unit spikes, before the FFT analysis, were subjected to digital, low-pass filtering performed by convolving the sequence of standardized pulses representing the spike trains with a sinc function [i.e. of the form $\sin (\pi \mathrm{t}) / \pi \mathrm{t}$ ] with parameters set so that the information in the spectra represented the interspike intervals rather than the shape of the standardized pulses.

\section{Results}

Neuronal activity of the brainstem tegmentum was sampled from 70 locations, representing different subdivisions of Gudden's nuclei. Histological examination revealed that 27 of these recordings were made in the anterior (ATg), 40 in the ventral (VTg, see example in Fig. 1B) and three in the dorsal (DTg) tegmental nuclei. Hippocampal field potentials and the neuronal discharge in each experiment were sampled during both theta and nontheta states. Theta states characterized by rhythmic synchronization of the hippocampal EEG (Fig. 1) appeared either spontaneously or were induced by sensory stimulation (i.e. by pinching the tail or paw or stroking the fur on the rat's back). Theta rhythm recorded during tail pinch was faster (4-4.5 Hz) and more stable than spontaneous theta $(3.2-3.8 \mathrm{~Hz})$. In most experiments, sensory-evoked, rhythmic slow activity did not terminate when the stimulation was discontinued but the oscillations became slower and similar to spontaneous theta waves.

\section{Firing rate}

The average firing rates of single cells ( $n=39$; multiunit recordings were not included in this analysis) during nontheta state (associated with low amplitude irregular activity in the hippocampus) were similar in the ATg and VTg $(24.3 \pm 2.7$ and $18.6 \pm 2.0$, respectively). Most neurons (31 out of 37) in these nuclei fired between 10 and 30 spikes/s and the two DTg neurons also fired within this range. 
FIG. 1. Synchronization of neuronal discharge in the midbrain tegmentum with rhythmic forebrain activity. Hippocampal electroencephalogram was recorded along with extracellular single-unit activity from the ventral tegmental nucleus of Gudden (VTg). (A) Schematic representation of the major anatomical connections linking Gudden's nuclei with the forebrain limbic system. (B) Reconstruction of the location of microelectrode's tip in VTg. DR, dorsal raphe; MR, median raphe. (C and D) Examples of hippocampal electroencephalogram (EEG) (upper traces) and VTg single-unit (lower traces) recordings. In response to sensory stimulation $(\mathrm{C})$, eliciting theta synchronization of hippocampal field potentials, the firing of VTg neuron switched from an irregular (D) pattern to regular theta rhythmicity. (E,F) Autocorrelogram of the VTg neuron discharge. $(\mathrm{G}$ and $\mathrm{H})$ Cross-correlation (spike-triggered averages) between neuronal discharge and hippocampal EEG.
A

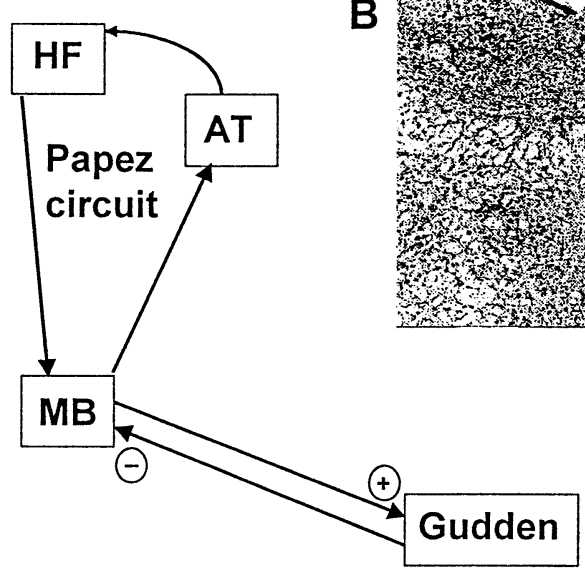

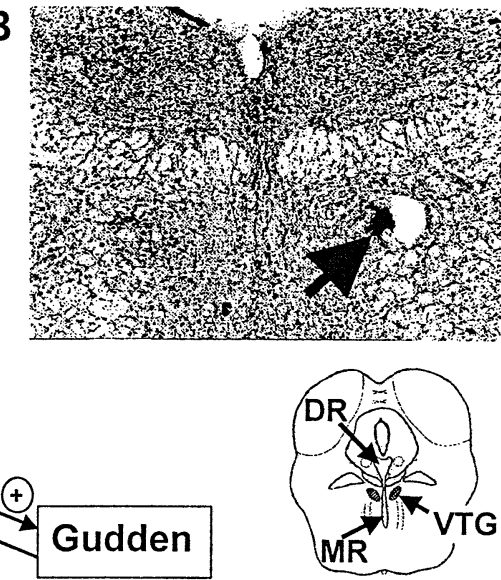
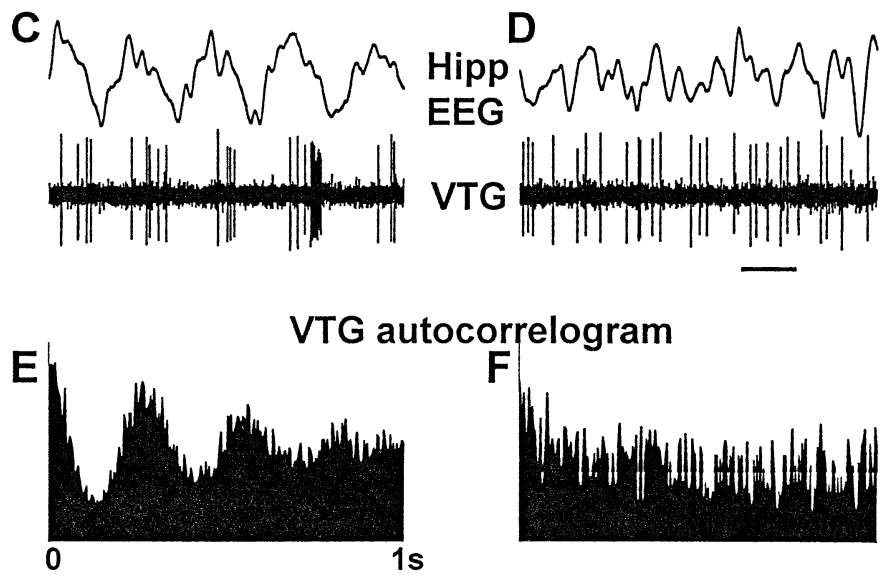

G

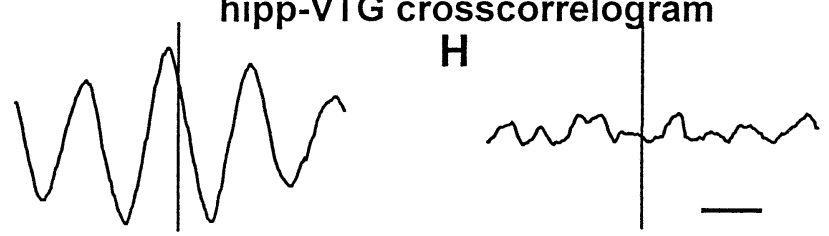

The discharge rate did not change during spontaneous theta (tested in eight ATg and nine VTg single-cell recordings) compared with the immediately preceding nontheta segment. Sensory stimulation, however, in addition to eliciting fast theta, produced a $20-125 \%$ increase in the firing rate of the majority of cells (22 of 33 tested) which then decreased as the stimulation ceased, even if hippocampal theta rhythm persisted. On average, sensory-elicited neuronal activation (increase in firing rate) was $48 \%$ in the $\operatorname{ATg}(P=0.003)$ and $19 \%$ in $\operatorname{VTg}(P=0.052)$.

\section{Theta rhythmic discharge in the nuclei of Gudden}

The firing characteristics of a typical VTg neuron and its relationship to hippocampal EEG is demonstrated in Fig. 1. During tail pinch (Fig. 1C, E and G) and during spontaneous theta activity in the hippocampus, the neuron fired rhythmic bursts at every theta cycle associated with the falling phase of field potential oscillations in the CA1 region. Accordingly, the cell's autocorrelogram, as well as the VTg-hippocampus cross-correlogram, both showed a large dominant rhythmic component at $3.7 \mathrm{~Hz}$. During the nontheta state (i.e. when the hippocampal EEG was desynchronized) (Fig. 1D, F and H), the neuron exhibited an irregular pattern of discharge with no significant peak in the autocorrelogram or in the spike-triggered average.

The rhythmic component in the EEG and neuronal discharge were also tested in the frequency domain (see sample autospectra in Figs 2-4) and the relationship between the two signals was quantified in each experiment using the coherence function (Fig. 2).

Rhythmic firing was observed in all recordings without exception. In fact, theta modulation of the discharge of cells densely packed (Petrovicky, 1973; Allen \& Hopkins, 1989; Hayakawa \& Zyo, 1991) in the ATg and VTg was so intense that it could always be readily detected as soon as the electrode reached the nucleus, even before spikes from single cells were clearly identified from the recording. The average coherence between theta rhythm in the hippocampal EEG and neuronal discharge in Gudden's nuclei was 0.60, 0.59 and 0.54 in the ATg, VTg and DTg, respectively. The coherence of multiunit recordings was somewhat lower than those calculated for single-unit spike trains, but the difference was not significant $(P=0.17)$. These estimates were computed using multiple theta segments (spontaneous as well as sensory elicited; $n=1-5$ for each unit) to increase statistical reliability. It should be noted, however, 
that they represent averages over time and in most cases underestimate the maximal strength of correlation that could be developed for shorter time intervals which were above 0.90 in $40 \%$ of all neuronal recordings.

\section{The dynamics of coupling between hippocampus and the Gudden nuclei}

Theta coherences measured during and after tail pinch were significantly different $(0.77+0.04$ and $0.62+0.04, P<0.0001)$, indicating that the strength of coupling changed during switching between these two states. Figure 2 shows two examples in which after the cessation of sensory stimulation, the theta rhythm appeared considerably stronger in one of the recordings than in the other, thus resulting in a decrease in the coherence between the two signals. During tail pinch, theta rhythm was clearly visible in both structures (Fig. 2A and B, upper traces) and produced sharp peaks in the hippocampal and VTg power spectra as well as in their coherence functions (Fig. 2C and D, left columns). After tail pinch, however, for the first comparison (Fig. 2A), strong theta synchronization continued in hippocampal EEG, although at a lower frequency, while neuronal discharge in ATg was less rhythmic. By contrast, for the second comparison (Fig. 2B) the VTg spike train was rhythmically modulated while irregular activity dominated the hippocampal EEG. Accordingly, a clear theta peak was only present in one of the autospectra in each case, while the other only contained a small hump at theta frequency (see arrows in Fig. $2 \mathrm{C}$ and D). The low peak in the averaged autospectrum indicated that the rhythmic component in one of the signals was either weak or not continually present. The coherence between hippocampus and $\mathrm{ATg} / \mathrm{VTg}$, however, was always significant at theta frequency indicating that when present in both structures the oscillations were strongly coupled.

To test whether temporal variations on a shorter time scale could explain lower coherence during spontaneous theta, we examined the time-frequency dynamics of rhythmic activity in the two signals, as demonstrated in a representative experiment in Fig. 3. In this rat, synchronous theta oscillations developed at $4.4 \mathrm{~Hz}$ during tail pinch in the hippocampus as well as in VTg, after which the power in both signals remained concentrated between 3 and $4 \mathrm{~Hz}$ for another 25-30 s (Fig. 3A and B). During this period, however, sharp peaks in hippocampal EEG autospectra were present for only a couple of seconds, at $3.8 \mathrm{~Hz}$ (see high peak at $57 \mathrm{~s}$ and a lower one at $47 \mathrm{~s}$ in Fig. 3C). In the VTg, rhythmic synchrony existed longer but the dominant frequency fluctuated between 3.8 and $3.5 \mathrm{~Hz}$, giving rise to a chain of four to five separate peaks in the time-frequency representation (Fig. 3F).

Time-frequency plots constructed for different neurons or even for the same neuron during different theta events were highly variable. They all shared, however, some common features. First, sensory stimulation always elicited highly coherent simultaneous theta rhythm in both hippocampal EEG and ATg/VTg unit recordings. Second, during theta events without sensory stimulation, the power in both signals was concentrated within the same, relatively narrow, frequency range. Third, on this background, short transient peaks often appeared synchronously in the two autospectra but many times peaks well developed in one signal were unaccompanied by similar peaks in the other signal. Fourth,
A hipp

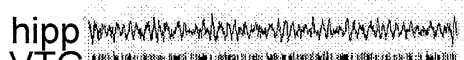

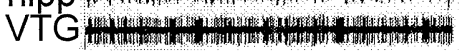

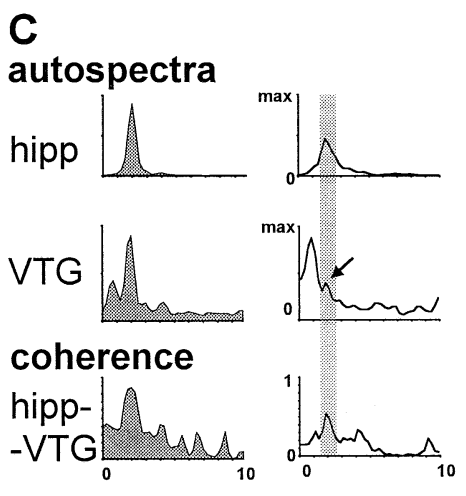

B

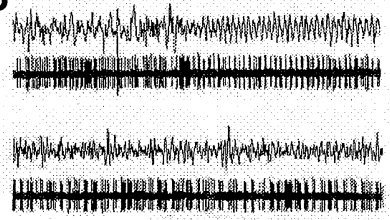

D

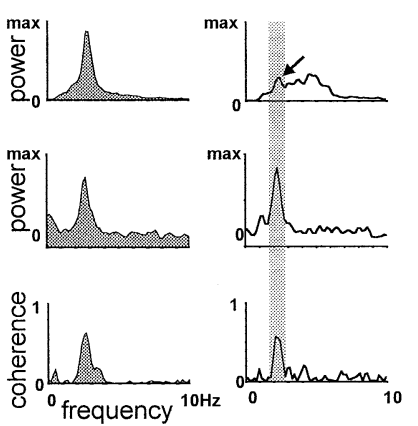

FIG. 2. Dissociation of rhythmic neuronal discharge in the brainstem tegmentum and theta field activity of the hippocampus. (A and B) Examples of coupling and uncoupling between neuronal activities in hippocampus (upper traces) and ventral tegmental nucleus of Gudden (VTg) (lower traces) in two rats; top: switch between irregular activity and theta rhythmicity in both structures on sensory stimulation; bottom: after the sensory stimulus ceased theta activity often remained either in the hippocampus (A) or VTg (B) or both (not shown). (C and D) Power spectra of hippocampal electroencephalogram (EEG) (top) and VTg firing (middle), and the coherence between the two signals. Note prominent theta peaks in all spectra during tail pinch (C and D, left columns) and contrasting patterns in the two experiments after tail pinch (C and $\mathrm{D}$, right columns). Note also significant coherence at theta frequency when theta only dominated one of the signals (i.e. hippocampus in C and VTg in D) and was scantily present in the other (see arrows).

FIG. 3. Time-frequency analysis of theta activity in the hippocampus and ventral tegmental nucleus of Gudden (VTg) during and after sensory stimulation. (A and B) Contour plot representing the temporal variations in hippocampal EEG and VTg unit discharge autospectra. During tail pinch, both structures exhibited theta rhythmic activity at $4.4 \mathrm{~Hz}$. After the sensory stimulus ceased, rhythmic activity remained in both structures, between 3.3 and $3.9 \mathrm{~Hz}$. (C and F) Time-frequency contour plot at higher resolution demonstrates two alternating rhythmic components in the VTg autospectrum, one at $3.5 \mathrm{~Hz}$ and the other at $3.8 \mathrm{~Hz}$. Only one of these (i.e. at $3.8 \mathrm{~Hz}$ ) was also present in the hippocampus. (D) Autospectra of three $4 \mathrm{~s}$ segments exhibiting rhythmic activity at $3.8 \mathrm{~Hz}$ simultaneously in the hippocampus and in VTg. (E) Autospectra of three other segments in which slow theta peak at $3.5 \mathrm{~Hz}$ was only present in the discharge of VTg. 

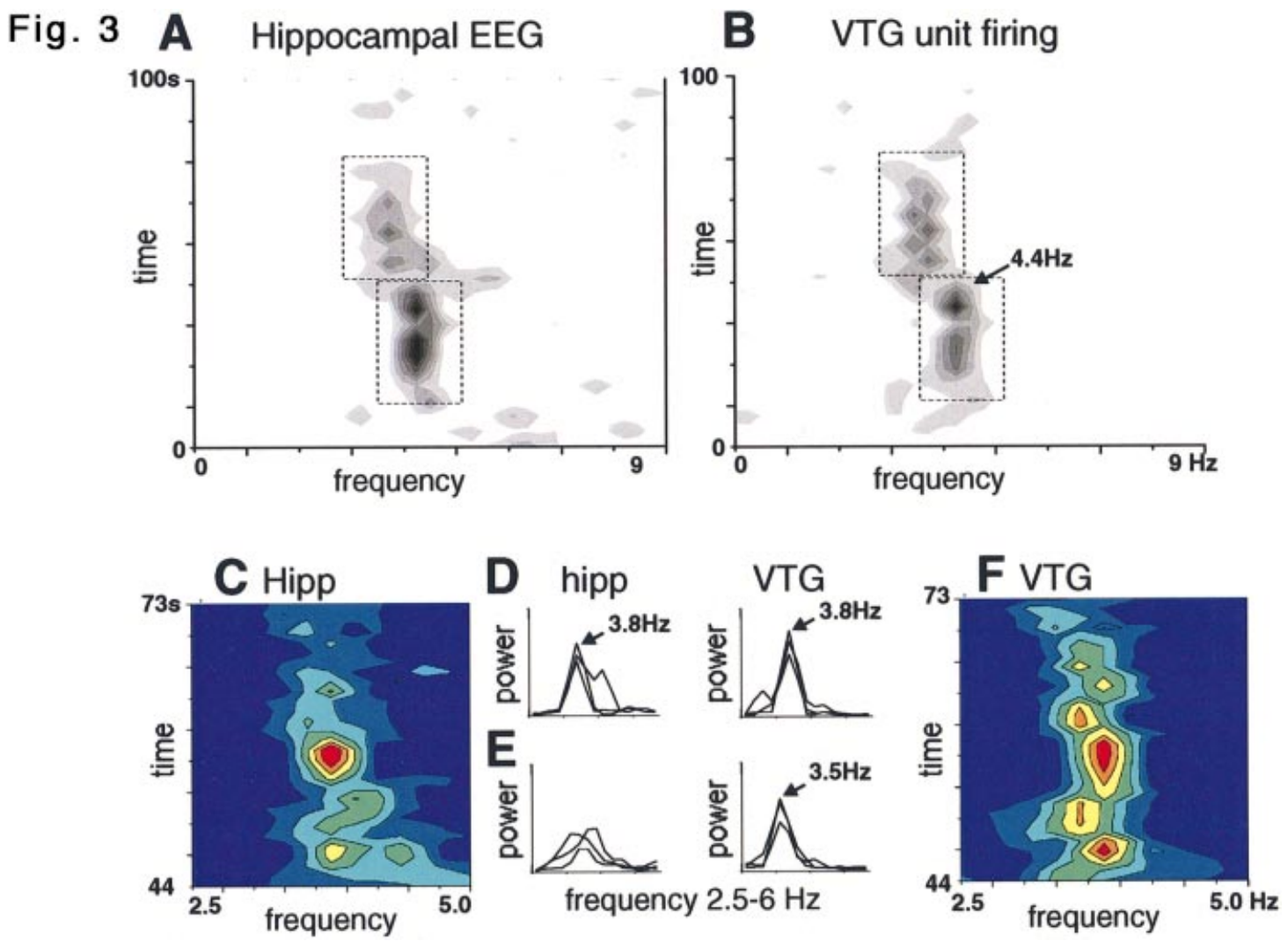

FIG. 3. Legend opposite.

FIG. 4. Dissociation of the onset of rhythmic activity in the hippocampus and ventral tegmental nucleus of Gudden (VTg) elicited by sensory stimulation. (A) The neuron in VTg (lower trace) switched from irregular to rhythmic firing immediately after the onset of sensory stimulus (see arrow), while

hippocampal electroencephalogram (EEG) (upper trace) demonstrated a relatively slow build up of rhythmic theta waves lasting about 2-3 s. (B and C) Time-frequency contour plot demonstrating the time development of rhythmic synchronization in the hippocampus and VTg. In both structures, theta started at $4.2 \mathrm{~Hz}$ and accelerated to $4.8 \mathrm{~Hz}$ as the amplitude increased. Note that not only did the onset of theta peak occur later in hippocampus than in VTg but its further development (i.e. changes in frequency and amplitude) lagged behind that in VTg. (D) Autospectra of three segments of hippocampal and VTg signals show no theta in the control recordings (1), sharp theta peak in VTg unparalleled in the hippocampus immediately after the onset of tail pinch (2) and simultaneous theta in both signals later (3).
Fig. 4
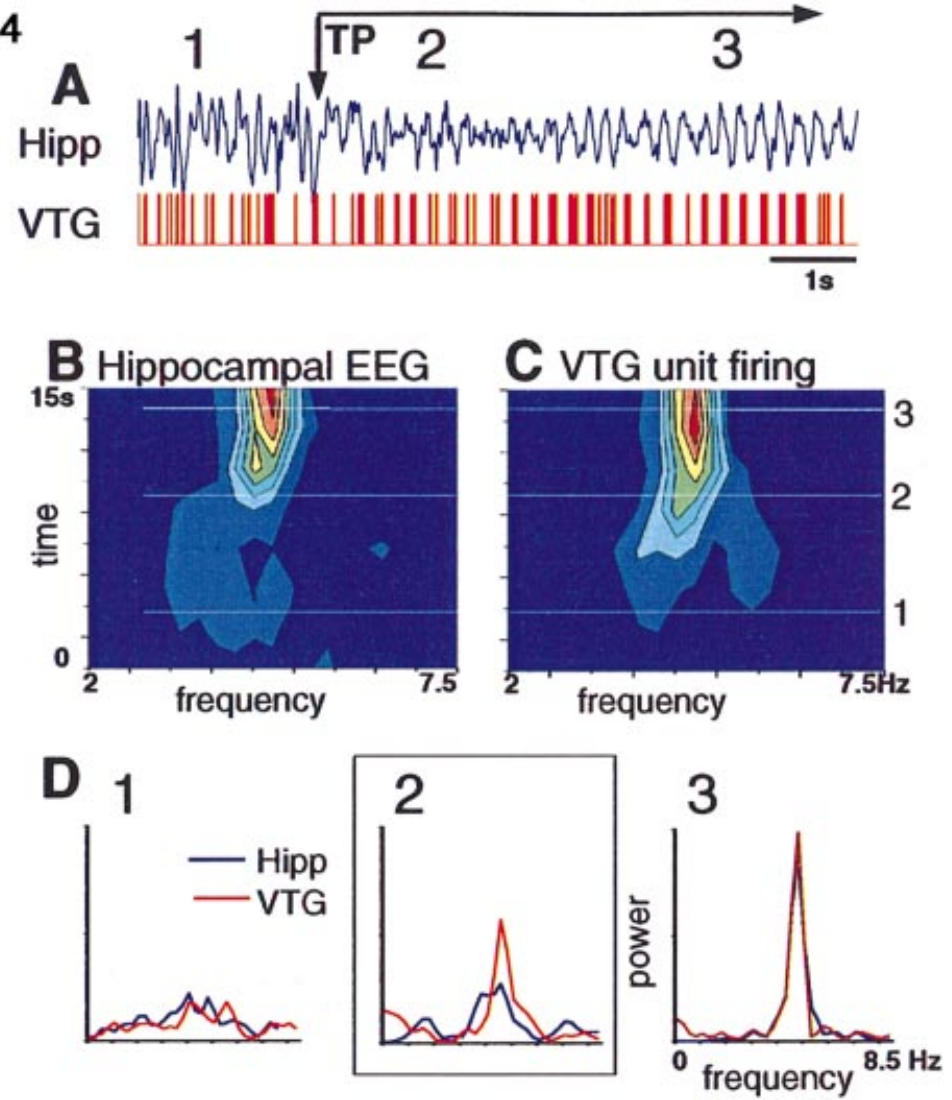
sharp spectral peaks never occurred simultaneously at different frequencies in the two autospectra.

\section{Onset of theta rhythm elicited by sensory stimulation}

Differences could also be seen in the onset of theta synchronization in the hippocampus and the Gudden nuclei. The traces in Fig. 4A are representative of eight experiments in which sensory-evoked synchronization of hippocampal field potentials required $2-3 \mathrm{~s}$ to fully develop, while rhythmic firing appeared immediately in the spike train of the VTg neuron. The asynchronous onset of the concentration of power at theta frequency was clearly documented in the frequency domain by the time-frequency plots of the two signals. Theta oscillations in the experiment in Fig. 4 started $\sim 2 \mathrm{~s}$ earlier in the VTg than in the hippocampus (at $6.3 \mathrm{~s}$ and $8.5 \mathrm{~s}$, respectively; note also sharp peak in the VTg but not in hippocampal EEG autospectrum at $9 \mathrm{~s}$, in Fig. 4D2). A variant of this pattern occurred in other experiments in which rhythmic waves appeared immediately in the hippocampal EEG as well, but they were of low amplitude first and increased a crescendo in the next 2-3 s until reaching the amplitude which was then maintained during the rest of the sensory stimulation.

\section{Discussion}

Although most prominent in the hippocampus, theta rhythmic activity (field potentials and/or neuronal discharge) have been recorded in a number of related forebrain structures, including the entorhinal cortex (Mitchell et al., 1982; Alonso \& Garcia-Austt, 1987), medial septum (Petsche et al., 1962), nuclei of the mammillary complex (Green \& Arduini, 1954; Komisaruk, 1970; Kirk \& McNaughton, 1991; Kocsis \& Vertes, 1994; Bland et al., 1995), anterior thalamus (Le Moal \& Cardo, 1975; Albo et al., 1999; Blair et al., 1999), basal forebrain (Manns et al., 2000) and basolateral amygdala (Pare \& Gaudreau, 1996; Heinbockel \& Pape, 2000). The major finding of the present study was that neuronal activity exhibiting strong state-dependent synchrony with the rhythmic hippocampal EEG is present also at the brainstem level, specifically in the relatively small tegmental nuclei of Gudden that are intimately connected with the limbic forebrain. Whereas the anatomy of Gudden's nuclei have been studied in much detail, to our knowledge, this is the first study to explore the discharge properties of neurons located in these nuclei. In accord with anatomical data (Petrovicky, 1973; Allen \& Hopkins, 1989; Hayakawa \& Zyo, 1991), our findings indicate homogeneous neuronal populations in the ATg and VTg; that is, the neurons fired at similar rates and all exhibited a consistent switch from irregular discharge to rhythmic bursts during theta states. The switch between these patterns closely matched the analogous transformations in the hippocampal EEG, but the level of synchrony between the two signals varied depending on the level of theta activation. During sensory stimulation, theta was faster and more regular, and the rhythmic bursts in the tegmentum showed extremely high coherence (up to 0.96 ) with hippocampal field potentials. During spontaneous theta, the oscillations in the two structures often dissociated and the average coherence was significantly lower.

Gudden's nuclei do not have direct connections with the hippocampus or the septal theta pacemaker. They are closely associated, however, with the MB which receives input from various sources exhibiting theta rhythmic activity. MB neurons have been shown to fire rhythmically during theta states (Komisaruk, 1970; Kocsis \& Vertes, 1994; Bland et al., 1995). There is a strong reciprocal link between the $\mathrm{MB}$ and tegmental nuclei of Gudden.
Quantitative ultrastructural study of MB afferents showed that $77.7 \%$ of all terminals with pleomorphic vesicles and symmetric contacts (i.e. presumed inhibitory synapses) in the medial MB originate from the VTg of Gudden (Hayakawa \& Zyo, 1991). These neurons appear to establish a strong recurrent inhibitory network in that the majority of $\mathrm{MB}$ neurons (78\%) targeted by VTg afferents was also retrogradely labelled from VTg. In turn, the majority of $\mathrm{MB}$ terminals contacting VTg neurons contains round vesicles and forms asymmetric contacts, suggesting that the $\mathrm{MB} \rightarrow \mathrm{VTg}$ projection is mainly excitatory. Thus, the MB-VTg network exhibits a number of similarities with other excitatory-inhibitory recurrent systems known to generate and maintain rhythmic oscillations at low frequencies (Contreras \& Steriade, 1996; Contreras et al., 1996; Ritz \& Sejnowski, 1997; Llinas et al., 1999; Plenz \& Kitai, 1999). Furthermore, many VTg neurons also express the neuropeptide neurotensin (Gonzalo-Ruiz et al., 1999) which may represent an additional mechanism supporting rhythmic firing behaviour in the MB. Activation of neurotensin receptors has been shown to promote oscillatory bursting in the basal forebrain (Alonso et al., 1994) and to increase limbic theta activity (Castel et al., 1989).

The synchrony of the MB-VTg oscillation with theta rhythm in the hippocampus (Komisaruk, 1970; Kocsis \& Vertes, 1994; Bland et al., 1995) can be influenced by descending inputs from the subiculum, entorhinal cortex and/or the MS. Procaine injections into the MS which reversibly suppress the theta pacemaker have been shown to abolish the rhythmic discharge of $\mathrm{MB}$ neurons and to severely attenuate their discharge rate (Kirk et al., 1996). This indicates that descending excitatory afferents to MB not only promote a coupling between the two rhythms but also provide sufficient excitation to initiate oscillatory behaviour in the MB-VTg recurrent network. Although the heaviest descending projection to MB originates from the subicular complex, the phasic signal for entrainment may involve septal neurons which, similar to cells in Gudden's nuclei, often begin firing rhythmic bursts before theta waves appear in the hippocampal EEG (Bland et al., 1999). The variability and the temporal fluctuation in the composition of the excitatory input and a rhythmic drive might explain the apparent dissociations between ATg/VTg and hippocampal oscillations seen in our experiments, and the dynamic variations in the strength of coupling. It should be emphasized, however, that in the present study the activity of the MS theta pacemaker was not monitored directly, but only through signals representing the activity of follower circuits. Significant coherence indicated a common origin of theta components in the hippocampus and ATg/VTg, but the two signals displayed different forms of this rhythm. In the hippocampus, we recorded field potential oscillations whereas single-unit activity was recorded in Gudden's nuclei. Although population activity in the hippocampus is organized by the theta rhythm, individual neurons may follow this rhythm with different intensities and regularities. On the other hand, temporal variations in Gudden's nuclei reflect the rhythm modified within the MB-tegmental resonant network.

Anatomical data strongly suggest that tegmental afferents play an important role in modulating the information flow from the hippocampal formation to the anterior thalamus, at the level of the MB. Descending fibres from the subicular complex arrive at the medial MB, topographically organized into horizontal laminae which are orthogonally orientated to the terminals formed by the tegmental input and to the MB neurons projecting to the thalamus (Allen \& Hopkins, 1989). The MB mainly contains projection cells which have branching axons with collaterals to both the anterior thalamus and to Gudden's nuclei. Significant computational capability of such a construction in a subloop of Papez's circuit has been demonstrated recently in a series of studies analysing the head direction circuit 
(Muller et al., 1996). The dorsal tegmental nucleus of Gudden is anatomically closely related to this particular circuit (Briggs \& Kaelber, 1971; Allen \& Hopkins, 1989; Hayakawa \& Zyo, 1990a). Regarding the MB-Gudden interaction in this network, Blair et al. (1998) proposed that the lateral inhibition between MB head direction cells, warranting their precise directional tuning, is routed through the DTg. Specifically, they proposed that this long loop through the brainstem, mediated by inhibitory actions of DTg cells on MB, serves to integrate vestibular input (Skaggs et al., 1995; Zhang, 1996; Blair et al., 1998) into the system responsible for the rat's sense of direction. Anatomical data suggest that VTg neurons might play an analogous role in the control of the subiculum $\rightarrow$ medial $\mathrm{MB} \rightarrow$ anteroventral thalamus circuit.

In addition to the interactions between neurons in the MB and tegmental nuclei, substantial 'escape pathways' (Nauta, 1958) radiate from the MB-Gudden loop, potentially channelling theta rhythmic activity from the $\mathrm{MB}$ to other brainstem nuclei, including the cholinergic (laterodorsal tegmental nucleus) and aminergic nuclei (locus coeruleus, dorsal and median raphe nuclei), and from the Gudden's nuclei to structures adjacent to $\mathrm{MB}$, such as the supramammillary, premammillary and histaminergic tuberomammillary nucleus (Allen \& Hopkins, 1989).

\section{Acknowledgements}

We thank Drs J. Allan Hobson and J. Quattrochi for valuable discussions. This work was supported by OTKA (T-17778, T-30344 to B.K.) and by NIH (MH 13913 to J.A. Hobson and NS 35883, MH01476 to R.P.V.).

\section{Abbreviations}

ATg, anterior tegmental nucleus of Gudden; CA, Ammon's horn; DTg, dorsal tegmental nucleus of Gudden; EEG, electroencephalogram; MB, mammillary body; MS, medial septum; VTg, ventral tegmental nucleus of Gudden.

\section{References}

Albo, Z., Viana Di Prisco, G. \& Vertes, R.P. (1999) Re-entrant theta activity in Papez circuit: role of the anterior ventral nucleus of the thalamus. Soc. Neurosci. Abstr., 25, 1407.

Allen, G.V. \& Hopkins, D.A. (1989) Mammillary body in the rat: topography and synaptology of projections from the subicular complex, prefrontal complex, and midbrain tegmentum. J. Comp. Neurol., 286, 311-336.

Allen, G.V. \& Hopkins, D.A. (1990) Topography and synaptology of mammillary body projections to the mesencephalon and pons in the rat. J. Comp. Neurol., 301, 214-231.

Alonso, A., Faure, M.P. \& Beaudet, A. (1994) Neurotensin promotes oscillatory bursting behavior and is internalized in basal forebrain cholinergic neurons. J. Neurosci., 14, 5778-5792.

Alonso, A. \& Garcia-Austt, E. (1987) Neuronal sources of the theta rhythm in the entorhinal cortex of the rat. II Phase relations between unit discharges and theta field potentials. Exp. Brain Res., 67, 502-509.

Alonso, A. \& Llinas, R.R. (1992) Electrophysiology of the mammillary complex in vitro. II. Medial mammillary neurons. J. Neurophysiol., 68 , 1321-1331.

Blair, H.T., Cho, J. \& Sharp, P.E. (1998) Role of the lateral mammillary nucleus in the rat head direction circuit: a combined single unit recording and lesion study. Neuron, 21, 1387-1397.

Blair, H.T., Cho, J. \& Sharp, P.E. (1999) The anterior thalamic head-direction signal is abolished by bilateral but not unilateral lesions of the lateral mammillary nucleus. J. Neurosci, 19, 6673-6683.

Bland, B.H., Konopacki, J., Kirk, I.J., Oddie, S.D. \& Dickson, C.T. (1995) Discharge patterns of hippocampal theta-related cells in the caudal diencephalon. J. Neurophysiol., 74, 322-333.

Bland, B.H., Oddie, S.D. \& Colom, L.V. (1999) Mechanisms of neural synchrony in the septohippocampal pathways underlying hippocampal theta generation. J. Neurosci, 19, 3223-3237.
Briggs, T.L. \& Kaelber, W.W. (1971) Efferent fiber connections of the dorsal and deep tegmental nuclei of Gudden. An experimental study in the cat. Brain Res., 29, 17-29.

Castel, M.N., Stutzmann, J.M., Lucas, M., Lafforgue, J. \& Blanchard, J.C. (1989) Effects of ICV administration of neurotensin and analogs on EEG in rats. Peptides, 10, 95-101.

Contreras, D., Destexhe, A., Sejnowski, T.J. \& Steriade, M. (1996) Control of spatiotemporal coherence of a thalamic oscillation by corticothalamic feedback. Science, 274, 771-774.

Contreras, D. \& Steriade, M. (1996) Spindle oscillation in cats: the role of corticothalamic feedback in a thalamically generated rhythm. J. Physiol. (London), 490, 159-179.

Gonzalo-Ruiz, A., Morte, L., Flecha, J.M. \& Sanz, J.M. (1999) Neurotransmitter characteristics of neurons projecting to the supramammillary nucleus of the rat. Anat. Embriol., 200, 377-392.

Green, J.D. \& Arduini, A. (1954) Hippocampal electrical activity in arousal. $J$. Neurophysiol., 17, 533-557.

Hayakawa, T. \& Zyo, K. (1990a) Fine structure of the lateral mammillary projection to the dorsal tegmental nucleus of Gudden in the rat. J. Comp. Neurol., 298, 224-236.

Hayakawa, T. \& Zyo, K. (1990b) Ultrastructure of the mammillotegmental projections to the ventral tegmental nucleus of Gudden in the rat. J. Comp. Neurol., 293, 466-475.

Hayakawa, T. \& Zyo, K. (1991) Quantitative and ultrastructural study of ascending projections to the medial mammillary nucleus in the rat. Anat Embriol., 184, 611-622.

Heinbockel, T. \& Pape, H.C. (2000) Input-specific long-term depression in the lateral amygdala evoked by theta frequency stimulation. J. Neurosci., 20, RC68.

Kirk, I.J. \& McNaughton, N. (1991) Supramammillary cell firing and hippocampal rhythmical slow activity. Neuroreport, 2, 723-725.

Kirk, I.J., Oddie, S.D., Konopacki, J. \& Bland, B.H. (1996) Evidence for differential control of posterior-hypothalamic, supramammillary, and medial mammillary theta-related cellular discharge by ascending and descending pathways. J. Neurosci., 16, 5547-5554.

Kocsis, B. (1999) Theta rhythmic discharge in the tegmental nuclei of Gudden dynamically coupled with hippocampal EEG. Soc. Neurosci. Abstr., 25, 1640.

Kocsis, B. \& Vertes, R.P. (1992) Dorsal raphe neurons: synchronous discharge with theta rhythm of the hippocampus in the freely moving rat. $J$. Neurophysiol., 68, 1463-1467.

Kocsis, B. \& Vertes, R.P. (1994) Characterization of neurons of the supramammillary nucleus and mammillary body that discharge rhythmically with the hippocampal theta rhythm in the rat. J. Neurosci., 14, 7040-7052.

Komisaruk, B.R. (1970) Synchrony between limbic theta activity and rhythmical behavior in rats. J. Comp. Physiol. Psychol., 70, 482-492.

Korsakoff, S.S. (1887) Disturbance of psychic function in alcoholic paralysis and its relation to the disturbance of the psychic sphere in multiple neuritis of non-alcoholic origin. Vestnik. Psichiatrii, 4, 2.

Le Moal, M. \& Cardo, B. (1975) Rhythmic slow wave activity recorded in the ventral mesencephalic tegmentum in the rat. Electroencephalogr. Clin. Neurophysiol., 38, 139-147.

Llinas, R.R. \& Alonso, A. (1992) Electrophysiology of the mammillary complex in vitro. I. Tuberomammillary and lateral mammillary neurons. $J$. Neurophysiol., 68, 1307-1320.

Llinas, R.R., Ribary, U., Jeanmonod, D., Kronberg, E. \& Mitra, P.P. (1999) Thalamocortical dysrhythmia: a neurological and neuropsychiatric syndrome characterized by magnetoencephalography. Proc. Natl. Acad. Sci. USA, 96, 15222-15227.

Mair, W.P.G., Warrington, E.K. \& Weiskrantz, L. (1979) Memory disorder in Korsakoff psychosis: a neuropathological and neuropsychological investigation of two cases. Brain, 102, 749-783.

Manns, I.D., Alonso, A. \& Jones, B.E. (2000) Discharge properties of juxtacellularly labeled and immunohistochemically identified cholinergic basal forebrain neurons recorded in association with the electroencephalogram in anesthetized rats. J. Neurosci, 20, 1505-1518.

Mitchell, S.J., Ranck, J.B.J., Steward, O. \& Olton, D.S. (1982) Medial septal area lesions disrupt theta rhythm and cholinergic staining in medial entorhinal cortex and produce impaired radial arm maze behavior in rats. $J$. Neurosci, 2, 292-302.

Muller, R.U., Ranck, J.B. \& Taube, J.S. (1996) Head direction cells: properties and functional significance. Curr. Opin. Neurobiol., 6, 196-206.

Nauta, W.J.H. (1958) Hippocampal projections and related neural pathways to the mid-brain in the cat. Brain, 81, 319-341. 
Pare, D. \& Gaudreau, H. (1996) Projection cells and interneurons of the lateral and basolateral amygdala: Distinct firing patterns and differential relation to theta and delta rhythms in conscious cats. J. Neurosci., 16, 3334-3350.

Petrovicky, P. (1973) Note on the connections of Gudden's tegmental nuclei. Acta Anat., 86, 165-190.

Petsche, H., Stumpf, C.H. \& Gogolak, G. (1962) The significance of the rabbit's septum as a relay station between the midbrain and the hippocampus. I. The control of hippocampus arousal activity by the septum cells. Electroencephalogr. Clin. Neurophysiol., 14, 202-211.

Plenz, D. \& Kitai, S.T. (1999) A basal ganglia pacemaker formed by the subthalamic nucleus and external globus pallidus. Nature, 400, 677-682.

Risold, P.Y. \& Swanson, L.W. (1996) Structural evidence for functional domains in the rat hippocampus. Science, 272, 1484-1486.

Ritz, R. \& Sejnowski, T.J. (1997) Synchronous oscillatory activity in sensory systems: new vistas on mechanisms. Curr. Opin. Neurobiol., 7, 536-546.

Shibata, H. (1989) Descending projections to the mammillary nuclei in the rat, as studied by retrograde and anterograde transport of wheat germ agglutinin-horseradish peroxidase. J. Comp. Neurol., 285, 436-452.

Skaggs, W.E., Knierim, J.J., Kudrimoti, H.S. \& McNaughton, B.L. (1995) A model of the neural basis of the rat's sense of direction. In Tesauro, G., Touretzky, D.S., Leen, T.K. (eds), Advanced Neural Information Processing Systems. MIT Press, Cambridge, MA, pp. 173-180.

Squire, L.S., Amaral, D.G. \& Press, G.A. (1990) Magnetic resonance imaging of the hippocampal formation and mammillary nuclei distinguish medial temporal lobe and diencephalic amnesia. J. Neurosci., 10, 3106-3117.

Stackman, R.W. \& Taube, J.S. (1998) Firing properties of rat lateral mammillary single units: head direction, head pitch, and angular velocity. J. Neurosci., 18, 9020-9037.
Swanson, L.W. \& Cowan, W.M. (1975) Hippocampo-hypothalamic connections: origin in subicular complex, not in Ammon's horn. Science, 189, 303-304.

Swanson, L.W. \& Cowan, W.M. (1979) The connections of the septal region in the rat. J. Comp. Neurol., 186, 621-665.

Sziklas, V. \& Petrides, M. (1998) Memory and the region of the mammillary bodies. Prog. Neurobiol., 54, 55070.

Tulving, E. \& Markowitsch, H.J. (1997) Memory beyond the hippocampus. Curr. Op. Neurobiol., 7, 209-216.

Vanderwolf, C.H. (1969) Hippocampal electrical activity and voluntary movement in the rat. Electroencephalogr. Clin. Neurophysiol., 26, 407-418.

Vertes, R.P. \& Kocsis, B. (1997) Brainstem-diencephalo-septohippocampal systems controlling the theta rhythm of the hippocampus. Neuroscience, $\mathbf{8 1}$, 893-926.

Victor, M., Adams, R.D. \& Collins, G.H. (1989) The Wernicke-Korsakoff Syndrome and Related Neurological Disorders Due to Alcoholism and Malnutrition. FA Davis, Philadelphia, PA.

Wernicke, C. (1881) Lehrbuch der Gehirnkrankheiten für Aerzte und Studirende, Vol. 2. Theodore Fischer, Kassel.

Winson, J. (1978) Loss of hippocampal theta rhythm results in spatial memory deficits in the rat. Science, 201, 160-163.

Wirtshafter, D. \& Stratford, T. (1993) Evidence for GABAergic projections from the tegmental nuclei of Gudden to the mammillary body in the rat. Brain Res., 630, 188-194.

Zhang, K. (1996) Representation of spatial orientation by the intrinsic dynamics of the head-direction cell ensemble: a theory. J. Neurosci., 16, 2112-2126. 